\section{Original Article}

Journal of Epilepsy Research pISSN 2233-6249 / elSSN 2233-6257

Received February 20, 2021

Revised May 11, 2021

Accepted May 16, 2021

Corresponding author:

Marcelo Bedoya-Sommerkamp, MD

Alberto Hurtado School of Medicine,

Universidad Peruana Cayetano Heredia, Av.

Honorio Delgado 430, San Martin de Porres,

Lima 15102, Perú

Tel. $+511-319-0000$

Fax. $+511-482-4541$

E-mail; marcelo.bedoya.s@upch.pe

\title{
Convulsive Status Epilepticus in a Cohort of Patients from a Peruvian Academic Hospital
}

\author{
Marcelo Bedoya-Sommerkamp, MD', Victor Hugo Chau-Rodríguez, MD, \\ Jesús Medina-Ranilla, MD' , Alejandro Escalaya-Advíncula, MD', Ray Ticse-Aguirre, MD', \\ Walter De La Cruz-Ramírez, MD², Jorge G Burneo, MD, MSPH ${ }^{3}$ \\ ${ }^{1}$ Alberto Hurtado School of Medicine, Universidad Peruana Cayetano Heredia, Lima; ${ }^{2}$ Instituto Nacional de Ciencias \\ Neurológicas, Lima, Perú; ${ }^{3}$ Epilepsy Program and Neuro-Epidemiology Unit, Schulich School of Medicine, \\ Western University, London, Canada
}

Background and Purpose: Status epilepticus is a neurologic emergency whose epidemiology, etiology and management are scarcely known in developing countries. Our objective was to describe the demographic and clinical features as well as the management of generalized convulsive status epilepticus (GCSE) in adult patients admitted to the emergency department of an academic hospital in Peru, between March 2019 and March 2020.

Methods: Observational study of a prospective cohort in which patients were assessed by the emergency and neurology department on the first day of hospitalization, at discharge and at 30 days post-discharge in a follow-up visit. Relevant demographics and clinical data were collected. After being encoded and sorted, univariate statistical analysis was carried out.

Results: Of the sample of 59 patients, $62.7 \%$ were males, $57.6 \%$ were unemployed, $89.8 \%$ did not finish high school, and $55.9 \%$ had intermittent GCSE with no seizure at arrival. The total calculated median times were: 60 minutes from GCSE onset to hospital arrival, 110 minutes from GCSE onset to 1st line therapy, and 7 minutes from hospital arrival to 1st line therapy. The most frequently used antiepileptic drugs were one dose of benzodiazepine (41.7\%), phenytoin $(76.9 \%)$, and additional doses of benzodiazepines $(60 \%)$ for $1 \mathrm{st}$, 2nd, and 3rd line therapies, respectively. The most frequent etiologies were antiepileptic drug suspension (27.1\%), undetermined (25.4\%) and acute stroke (11.8\%). $62.71 \%$ had 0-2 modified Rankin score at discharge.

Conclusions: In this cohort of patients, GCSE was mainly intermittent. Management times differed from the guidelines' recommendations. (2021;11:83-92)

Key words: Epilepsy, Status epilepticus, Peru

\section{Introduction}

Status epilepticus (SE) is a persistent epileptic seizure due to failure of cessation mechanisms or persistence of activation mechanisms, which induce abnormally prolonged seizures. ${ }^{1}$ Most SE cases (45-75\%) present as generalized convulsive status epilepticus (GCSE). According to the International League Against Epilepsy (ILAE), GCSE is characterized by a convulsion, defined as "episodes of abnormal bilateral muscle contractions, which could be sustained or interrupted", and associated with impaired consciousness for at least 5 minutes of duration. ${ }^{1}$ Although its annual incidence rate is low (12.6 per 100,000 person-years), SE is a medical and neurological emergency with high morbidity (20-50\%) and mortality rates (3-40\%). As such, timely diagnosis and proper treatment have proved to be the best strategies to reduce morbidity and mortality in SE. ${ }^{2,3}$

Information on epidemiology, etiology, management and prognosis of SE comes mainly from developed countries and, moreover, there are obvious differences regarding the epidemiological profile and treatment opportunities between developed and developing countries. In developing countries like Peru, there is limited information available on SE. ${ }^{4-7}$ Therefore, there is an urge for knowl- 
edge on demographic data, clinical presentation, etiologies, and prescribed treatments in each geographic area. Around $32.45 \%$ of the Peruvian population is located in Lima, the capital of the country, and therefore the description of a cohort of patients located here could be representative for the entire population. Furthermore, to date, few studies have recorded time-to-treat for each line of therapy even though it is one of the most important factors in the management of $\mathrm{SE}^{5,8}$ since irreversible neurological damage would occur after $30 \mathrm{mi}-$ nutes of SE. ${ }^{1,9}$ The present study aims to describe the characteristics and management of GCSE in a cohort of adult patients treated in the emergency department (ED) of an academic hospital in Lima, Peru. We conducted a factual description of the data and how epilepsy is managed according to resources and based on national recommendations.

\section{Methods}

This was an observational study of a prospective cohort of adult patients with GCSE from an academic hospital (Cayetano Heredia Hospital) in Peru, which is the referral center of the northern area of the capital city (Lima). All patients older than 18 years old admitted to the ED, with the diagnosis of GCSE between March 2019 and March 2020, were included. GCSE was defined either as a continuous generalized convulsive seizure with at least 5 minutes of duration witnessed by healthcare professionals (continuous generalized convulsive status epilepticus, CGCSE) or intermittent convulsive seizures with no regaining of consciousness in between, lasting for at least 5 minutes, with the last event witnessed by healthcare professionals (intermittent generalized convulsive status epilepticus, IGCSE). Once we recorded the data and noted certain blatant differences, we found it relevant to further divide this latter group in IGCSE with convulsive seizures on arrival at the ED and IGCSE without convulsive seizures on arrival. Patients transferred from another institution or those who received treatment outside ED were not included, as well as those with suspicion of nonconvulsive SE following a convulsive SE without recurrence of convulsions. Due to the nature of $S E$, the informed consent was initially obtained from the legal representative and, if possible, later from the patient.

Demographic data, clinical information including the history of epilepsy, GCSE presentation (continuous or intermittent), status epilepticus severity score (STESS), and modified Rankin score were obtained. Information regarding times between 1) GCSE onset and admission to ED (pre-hospital time), 2) ED admission and administration of the first-line antiepileptic drug (AED) (time of action), 3) GCSE onset and first AED administered (pre-therapeutic time), and 4) 1st to the 2nd line and 2nd to 3rd line AED administered (if needed) were recorded by our neurology team. We also recorded the total SE time, considering that the ending of SE was determined by recovery of consciousness, due to lack of electroencephalogram (EEG) in our ED (Fig. 1). To simplify data presentation and analysis, all minutes were rounded to integers.

Etiology was determined by checking the medical records, including neurologic consultations, and it was classified according to the ILAE ${ }^{1}$ as acute symptomatic, remote symptomatic, progressive symp-

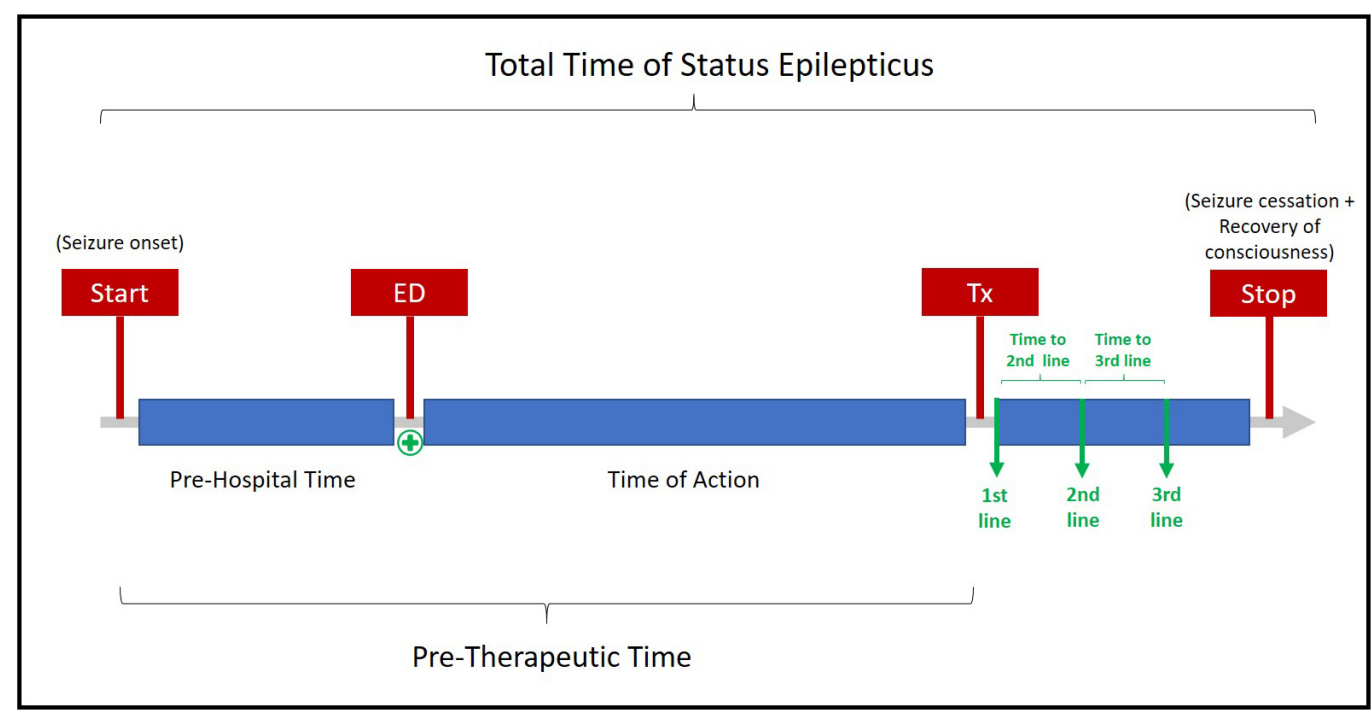

Figure 1. Measured times used in our study. ED, emergency department; Tx, pharmacological treatment administration. 
tomatic and unknown (not defined although all pertinent diagnostic tests were performed). Noteworthily, as this study was conducted in a low-resource country, we added "undetermined" (all pertinent diagnostic tests to define etiology could not be performed) to the etiology classification according to previously reported literature. ${ }^{5}$ Complications during hospitalization were recorded. Modified Rankin score was determined at hospital discharge and at 1-month follow-up. STATA ${ }^{\circledR} 15$ for Windows (StataCorp LLC, College Station, TX, USA) was used for data analysis. After quality control was performed on data entry, univariate analysis was carried out. For quantitative values, median, range and interquartile range were calculated, while for qualitative values, proportions were calculated. Because we did not calculate a sample, but included the entire population of patients who met our inclusion criteria during the recruitment phase, statitistical significance was not calculated. The study was approved by both the Ethics Committees from Universidad Peruana Cayetano Heredia and Cayetano Heredia Hospital.

Table 1. Clinical and demographic characteristics

\begin{tabular}{lc}
\hline Variable & Total (n=59) \\
\hline Age (years) & $47(18-92)$ \\
Sex & $37(62.7)$ \\
Male & \\
Occupation & $34(57.6)$ \\
Unemployed & $25(42.4)$ \\
Student/employed & \\
Educational level & $53(89.8)$ \\
Did not finish high school & $4(6.8)$ \\
Finished high school & $2(3.4)$ \\
People with special abilities & \\
Place of origin & $32(54.2)$ \\
Northern area of Lima & $27(45.8)$ \\
Other areas of Lima & \\
STESS & $30(50.9)$ \\
$<3$ & $31(52.5)$ \\
Previous diagnosis of epilepsy & $14(23.7)$ \\
Previous SE & \\
Clinical presentation of SE & $11(18.6)$ \\
CGCSE & $15(25.4)$ \\
IGCSE with convulsive seizures on arrival & $33(55.9)$ \\
IGCSE with no convulsive seizures (no clinical \\
seizures) on arrival
\end{tabular}

Values are presented as median (range) or number (\%). STESS, status epilepticus severity score; SE, status epilepticus.

\section{Results}

Fifty-nine patients met our inclusion criteria and were enrolled. Additionally, three patients received pre-hospital treatment and one was transferred from another institution, which were excluded. The median age was 47 years; most were male (62.7\%), unemployed (57.6\%), from the northern area of Lima, (54.2\%), and $89.8 \%$ of them did not finish high school. Regarding presentation of $S E$, $55.9 \%$ (33 cases) had IGCSE without convulsive seizures at arrival, while $25.4 \%$ (15 cases) had IGCSE with convulsive seizures at arrival; only $18.6 \%$ (11 cases) presented with continous seizure activity of CGCSE (Table 1). $94.9 \%$ (56 cases) had some comorbidity at arrival, with most of them having more than one.

The median total GCSE time was 120 minutes (range, 5-2,758) and 30 minutes (range, 6-340) for CGCSE, while for IGCSE, those who were convulsing at arrival had a median time of 99 minutes (range, 20-240) and it was 190 minutes (range, 5-2,758) for those who were not. The overall median prehospital time was 60 minutes, 20 minutes (range, 5-60) for CGSE and 73.5 minutes (range, 2-719) for IGCSE. The overall median pre-therapeutic time was 110 minutes, 20 minutes in CGSCE (range, 6-60), 90 minutes (range, 20-240) in IGCSE with convulsive seizures at arrival, and 190 minutes (range, 30-1,171) in IGCSE without convulsive seizures at arrival (Fig. 2). The median time of action was 7 minutes (range, 0-571), <1 minute (range, 0-5) for continuously seizing patients, $<1$ minute (range, 0-3) among IGCSE patients with convulsive seizures at arrival, and $70 \mathrm{mi}-$ nutes (range, 1-571) among those without convulsive seizures at arrival (Fig. 2).

There were 36 cases of GCSE that ceased with 1st line AEDs (57.6\%), of which 29 ceased in $<1$ minute, five ceased in 5-15 minutes, and two ceased in 30 and 83 minutes. For the remaining 23 cases that did not cease, the median time to 2 nd line treatment was 20 minutes (range, 0-180). Time ranges for each of the three groups (CGCSE, IGCSE with and without convulsions at arrival) differed: 5-40 minutes, 5-55 minutes, and 0-180 minutes, respectively. Thirteen cases of GCSE ceased in 0 minutes with 2nd line AEDs $(22 \%)$ and for the remaining 10 cases, the median time to $3 \mathrm{rd}$ line treatment was 8.5 minutes (range, 0-651); 5 minutes (range, 0-10) in CGCSE, 2.5 minutes (range, 1-4) in IGCSE with convulsions at arrival and 95 minutes (range, 0-651) in IGCSE without convulsions at arrival (Fig. 2).

The most used AED overall was intravenous (IV) diazepam (48.1\%), followed by intramuscular (IM) midazolam (26.7\%). The 

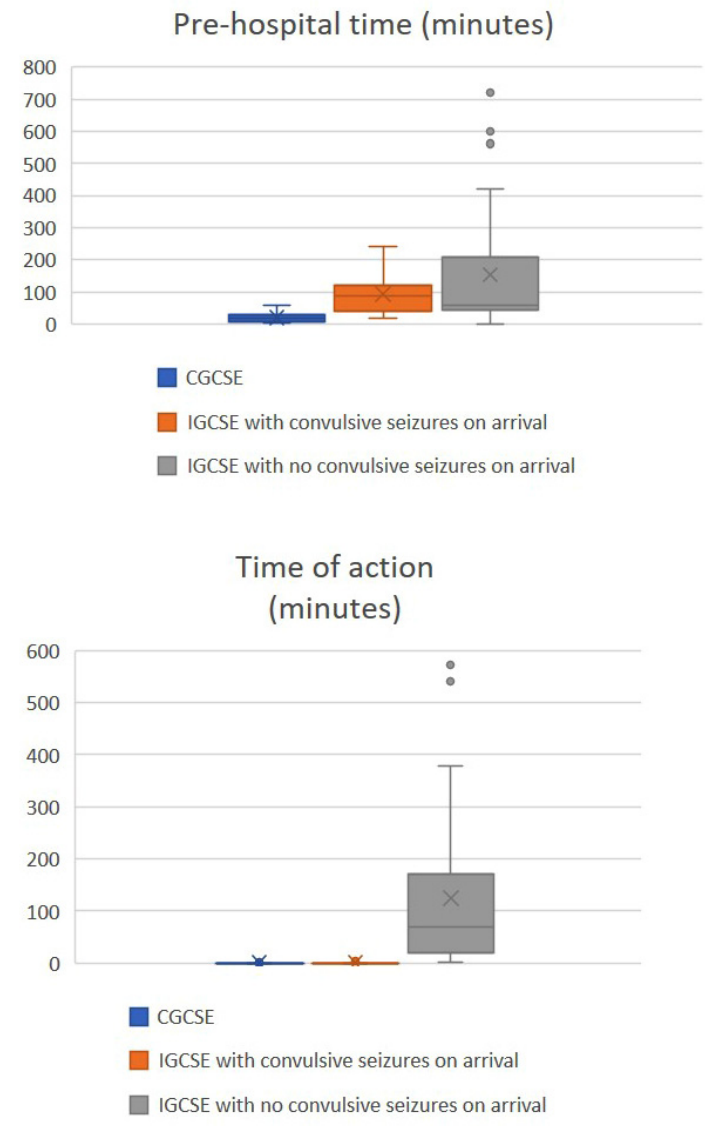

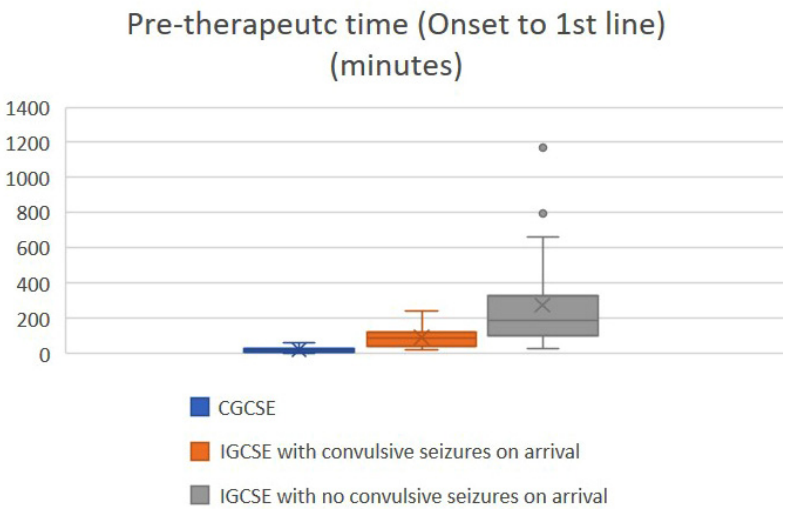

Registered times to 2nd \& 3rd Therapy lines (minutes)

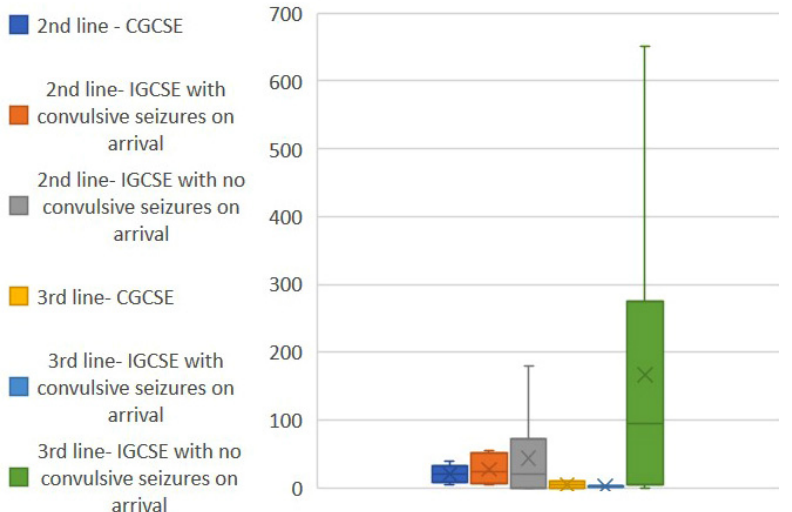

Figure 2. Overview of the times found in our study. CGCSE, continuous generalized convulsive status epilepticus; IGCSE, intermittent generalized convulsive status epilepticus.

most used AED as 1st line was a single-dose benzodiazepine (BZD) $(41.7 \%)$, as 2 nd line was phenytoin (76.2\%) and as 3rd line was the administration of an additional BZD (60\%) (Table 2). The adequate doses for each AED were calculated according to the current guidelines (11-14) and the estimated weight of each patient. In our study, only $56.6 \%$ of AEDs were administered at recommended doses. IV Diazepam was given in adequate doses 61 times (97\%); phenytoin loading dose was adequate eight times (36\%) and as 2nd dose it was adequate in three cases (100\%); propofol was given in adequate doses in two cases (100\%); IM midazolam was administered in insufficient doses 32 times (91\%), and phenytoin seven times (32\%); IV midazolam infusion was used three times (100\%) with superior doses than recommended.

As for GCSE etiology, it was undetermined in 15 patients (25.4\%), and unknown in two (3.4\%). Thirty-one patients (52.5\%) presented with an acute symptomatic etiology (AEDs suspension and acute stroke being the most frequent), nine patients (15.3\%) with a remote symptomatic etiology (chronic alcohol intake, metabolic disorders and previous history of traumatic brain injury [TBI] being the most frequent), and two patients (3.4\%) had a progressive symptomatic etiology (one had metastatic melanoma and the other, toxoplasmosis). Among all patients in our study, only three (5.1\%) had an infectious etiology (neurocysticercosis [NCC], toxoplasmosis). Differences regarding GCSE etiologies among patients with epilepsy were found (Table 3). From the diagnostic work-up performed to define GCSE etiologies, 10 out of 28 patients (35.7\%) who needed a lumbar puncture had the procedure done. On the other hand, 44 out of 59 patients $(74.6 \%)$ had a computed tomography (CT) scan, whereas only six (10.2\%) had also an magnetic resonance imaging (MRI). Twelve out of 16 patients with an undetermined etiology had a CT scan, but only one had an MRI. None of the enrolled patients had an EEG at the ED.

In-hospital complications appeared in 33 patients (55.9\%). The most frequent complications were: intrahospital/aspiration pneumo- 
Table 2. SE remission by line of therapy

\begin{tabular}{|c|c|c|c|}
\hline AEDs & Total & Achieved remission & SE remission (\%) \\
\hline 1st line & 59 & 36 & 61.0 \\
\hline 1 benzodiazepine & 26 & 17 & 65.4 \\
\hline 2 benzodiazepines & 12 & 6 & 50.0 \\
\hline >2 benzodiazepines & 4 & 0 & 0.0 \\
\hline Phenytoin (loading dose) & 15 & 12 & 80.0 \\
\hline Phenytoin (loading dose + 2nd dose) & 2 & 1 & 50.0 \\
\hline 2nd line & 23 & 13 & 56.5 \\
\hline Phenytoin (loading dose) & 16 & 10 & 62.5 \\
\hline Phenytoin (loading dose + 2nd dose) & 1 & 0 & 0.0 \\
\hline Additional benzodiazepines & 5 & 2 & 40.0 \\
\hline Midazolam (infusion) & 1 & 1 & 100.0 \\
\hline 3rd line & 10 & 10 & 100.0 \\
\hline Propofol & 1 & 1 & 100.0 \\
\hline Phenytoin (loading dose) & 0 & 0 & 0.0 \\
\hline Additional benzodiazepines & 6 & 6 & 100.0 \\
\hline Magnesium Sulphate & 1 & 1 & 100.0 \\
\hline Ketamine + benzodiazepines & 1 & 1 & 100.0 \\
\hline Propofol + benzodiazepines & 1 & 1 & 100.0 \\
\hline Total & 59 & 59 & 100.0 \\
\hline
\end{tabular}

Values indicate number of patients.

SE, status epilepticus; AEDs, antiepileptic drugs.

Table 3. GCSE etiologies

\begin{tabular}{lccc}
\hline \multirow{2}{*}{ Etiology } & \multicolumn{2}{c}{ Epilepsy history } & Total \\
\cline { 2 - 4 } AED's suspension & Yes & $0(0.0)$ & $16(27.1)$ \\
Undetermined & $16(27.1)$ & $12(20.3)$ & $15(25.4)$ \\
Acute stroke & $3(5.1)$ & $6(10.1)$ & $7(11.8)$ \\
Metabolic disorder* & $1(1.7)$ & $3(5.1)$ & $3(5.1)$ \\
CNS neoplasm & $0(0.0)$ & $2(3.4)$ & $3(5.1)$ \\
CNS infection & $1(1.7)$ & $1(1.7)$ & $3(5.1)$ \\
Alcohol withdrawal & $2(3.4)$ & $1(1.7)$ & $3(5.1)$ \\
Chronic alcoholism & $2(3.4)$ & $0(0.0)$ & $3(5.1)$ \\
Unknown & $3(5.1)$ & $1(1.7)$ & $2(3.4)$ \\
Others & $1(1.7)$ & $2(3.4)$ & $4(6.8)$ \\
Total & $2(3.4)$ & $28(47.5)$ & $59(100.0)$ \\
\hline
\end{tabular}

Values are presented as number (\%).

GCSE, generalized convulsive status epilepticus; AED, antiepileptics drug; CNS, central nervous system.

*Uremic encephalopathy, hyponatremia, hypocalcemia.

'Metastatic melanoma, unspecified neoplasm.

Neurocysticercosis, toxoplasmosis.

${ }^{\S}$ Traumatic brain injury, recreational drug withdrawal, cranial surgery postoperative.

nia (15.3\%), metabolic acidosis (15.3\%), and intracranial hypertension (8.5\%). Other common complications were electrolyte abnormalities (including sodium, potassium, magnesium, calcium, phosphate and glucose) and psychiatric complications (psychomotor agitation, BZDs' intoxication, alcohol withdrawal syndrome). Two patients were admitted to the intensive care unit (ICU). The median 
hospital stay was 4 days (range, 1-28). Regarding patients' functional state at discharge, $62.7 \%$ had a low modified Rankin score (0-2, asymptomatic-slight disability) and $28.8 \%$ of them were discharged with moderate/severe disability (Rankin score, 3-5). Five patients died during hospitalization (8.5\%) and all of them had IGCSE without convulsions at arrival. Finally, at 1-month after discharge, $49.2 \%$ of patients had a follow-up outpatient consultation. Among those who had a modified Rankin score $0-2,43.2 \%$ returned for follow-up, and $76.5 \%$ of those with moderate/severe disability returned. During the first month after discharge, one patient died of causes unrelated to epilepsy (metastatic melanoma).

\section{Discussion}

\section{Demographics}

In our population, most patients were male and had previous history of epilepsy, similar to previously reported guidelines. ${ }^{5}$ Although literature is scarce on information regarding the clinical presentation of GCSE as continuous or intermittent, the latter is reported as the most frequent, ranging from $30.5 \%$ to $65.8 \%{ }^{5,10-12}$ This resembles the data obtained from our cohort (81.4\%). In our study, total GCSE time was higher for IGCSE than CGCSE. One study found that it was 2.5 more likely for IGCSE to last as compared to CGCSE because of delays in making emergency calls or due to the lack of acknowledgement of SE as an emergency by the patients' relatives and companions. ${ }^{11}$ In our context, a Peruvian study found a direct relationship between educational level and knowledge of epilepsy and seizures, ${ }^{13}$ which could explain the delays in patients' arrival to hospitals due to both the lack of education, regarding identification and first-aid of seizures, and the lack of social support for patients with epilepsy, due to the ongoing social stigma that forces them to hide their condition.

\section{Pre-hospital time}

The overall delay in admission to the ED (pre-hospital time) is likely due to deficiencies in the healthcare system, connectivity and delays in transport, more predominant in developing countries compared to developed ones. ${ }^{12,14}$ In our study, the delay in admission to ED was also different between those with CGCSE and IGCSE. It is worth noting that in high-income countries an important number of patients receive treatment from emergency services before reaching the $E D^{15}$ which is rather uncommon in our setting as only three patients were excluded from our cohort for that reason. Patients with CGCSE ar- rived faster at the ED, possibly because an ongoing convulsion represents an alarming and unusual occurrence (particularly in patients with previous history of epilepsy, known seizures types and post-ictal phases), which ultimately compels relatives and companions to act quickly. On the other hand, patients with IGCSE arrived much later compared to patients with CGCSE. This might be because relatives and companions were less alarmed when they saw the patient without response or movement after the first convulsion, assuming this condition would subside on its own (appealing to the lack of information on seizures and epilepsy in the general population), or that it constituted their usual postictal phase (in patients with epilepsy), and thus they only felt the urge to take them to the ED after significant time had passed without recovery.

\section{Time of action}

The delay from ED admission to the first AED administered (time of action) depends on the staff and the institution taking care of the patient. Nevertheless, in our study, we observed that it also depends on the clinical presentation of GCSE. Most of the patients presenting with IGCSE arrived at ED only with impaired consciousness (non-convulsive phase), and they needed to have a convulsion again in order to receive adequate diagnosis and treatment, resulting in an increased delay in treatment compared to those with CGCSE. It is worth noting that after cessation of the GCSE motor manifestations, non-convulsive status epilepticus (NCSE) may ensue, with or without subtle motor manifestations. ${ }^{5,16-18}$ In these situations, it would be recommended to perform an EEG on arrival at the ED for timely diagnosis and treatment. ${ }^{3,5,19,20}$

\section{Treatment times}

In regard to treatment, it has been shown that adequate treatment has an impact on prognosis. ${ }^{10,21}$ Guidelines recommend 1st line therapy at 5 minutes of GCSE onset. ${ }^{8,22,23}$ Some studies report a median delay from 20 minutes to several hours for the start of therapy, ${ }^{10,12}$ while in our study we evidenced a delay to treatment (pre-therapeutic median time) of 110 minutes. Also, many studies have shown the difficulty in following guidelines' recommended treatment administration times due to factors such as lack of pre-hospital management, long distances to healthcare institutions and delays in using the emergency services, ${ }^{11,24,25}$ which are also shown in our study. In addition, a review concluded that only SE that started in the ICU followed recommended management times. ${ }^{10}$ 
Another study on refractory GCSE in the pediatric population found that both out-of-hospital onset and IGCSE were independently associated risk factors for delays to first AED administration. Thus, emphasis on pre-hospital management is encouraged by the authors of this study ${ }^{12}$ as well as by newer guidelines. ${ }^{22}$

\section{Time to 1st line treatment}

Regarding 1st line AED, guidelines recommend BZDs (evidence level A) ${ }^{8,22,23}$ IV lorazepam is considered first-choice for its short latency time and longer half-life time. ${ }^{8,23}$ Even though IV lorazepam is not available in Peru, it has been shown that IV (e.g., lorazepam, diazepam) and IM (e.g., midazolam) BZDs are equivalent in effectiveness. ${ }^{5,10}$ In our study, most of the patients received BZDs. Even though most studies report time measurements from SE onset to the administration of 2nd and/or $3 \mathrm{rd}$ line therapies, we considered that the measurement of the delays in-between management lines would be more useful due to their dependence on in-hospital factors, particularly those regarding healthcare staff management, and not on the out-hospital factors discussed above.

\section{Time to 2nd line treatment}

It is recommended to initiate 2 nd line therapy within 20 minutes of GCSE onset or within 15 minutes after 1st line therapy, given a lack of response (persistence of the $\mathrm{SE})_{i}^{8,23}$ in our setting, however, due to lack of $E E G$, 2nd line was initiated given persistence or reappearance of a clinically evident convulsion. In the present study, the median time between 1st and 2nd line was 20 minutes, exceeding by 5 minutes the guidelines' recommendations. To date, there is no evidence for effectiveness superiority of any AED over another (e.g., phenytoin over levetiracetam) as 2nd line therapy. ${ }^{8,10,23}$ In our study, more than half of the patients received IV phenytoin, a drug preferred in our hospital due to lower risk of respiratory depression compared to other AEDs used as 2nd line therapy (e.g., phenobarbital), although a code cart and serun dosage monitoring are required for its administration, due to associated cardiotoxicity. ${ }^{26}$ Other AEDs such as levetiracetam or valproic acid were not available at our hospital.

\section{Time to 3rd line treatment}

Third line treatment is usually administered 40 minutes after the onset of GCSE or 20 minutes after administering 2nd line therapy if there is no response. ${ }^{8,23}$ In the literature it is reported a total time of 2-3 hours between SE onset and the administration of 3rd line treatment, ${ }^{15}$ how- ever, the time between 2nd and 3rd line therapy has not been previously described. In our study, a median of 8.5 minutes was observed between 2nd and 3rd line therapies; nevertheless, there was a difference between the median of CGCSE and IGCSE. This may be due to the haste to treat patients with continuous seizures, or the difficulty in determining the response to treatment due to lack of EEG when the seizures had ceased and there was no full recovery of consciousness in IGCSE. Thus, it is recommended to perform an EEG to determine the true cessation of SE in these scenarios. ${ }^{5,20}$ As for 3rd line therapy, IV anesthetics (eg. propofol, thiopental, ketamine) and infusion BZDs (eg. midazolam) (level of evidence C-D) are indicated, along with continuous EEG (cEEG) monitoring for validating SE cessation. ${ }^{8,23}$ In our study, most of the patients who needed 3rd line treatment received additional doses of IV BZDs. This may be explained because anesthetics must be administered with continuous monitoring of vital functions (risk of hypotension and respiratory depression), anesthesiology consultation, and CEEG, preferably in the ICU. These resources were not readily available in our hospital, and therefore available BDZs were used. However, some studies have reported that the type of drug does not influence as much on the outcome or mortality of patients as the timing of therapy administration. ${ }^{10}$

\section{AED dosage}

Barely half of the patients received adequate AED doses. The infra and overdoses evidenced may be explained by a poor estimation of the patient's weight, the lack of knowledge of recommended doses and/or poor implementation of guidelines. ${ }^{8,23}$ On the other hand, the presentation of medications could also influence on its correct administration (e.g., in our hospital, midazolam vials contain $5 \mathrm{mg}$, whereas the recommended dose for adults is $10 \mathrm{mg}$ ), and poor response resulted from inadequate doses can be misinterpreted as refractoriness to treatment. ${ }^{10}$ This is especially important when there is no EEG available to ensure GCSE cessation, which could lead to an unnecessary change in management. ${ }^{19,20}$ In addition to the timing of management and the drugs used, the remission of GCSE also depends on the timely identification and management of its etiology (e.g., metabolic disorders associated SEs do not usually cease until the underlying cause has been treated). ${ }^{5,8,22,23}$

\section{Etiologies}

Previous Peruvian studies reported that the most frequent etiologies in our setting were AED suspension, acute symptomatic due to 
infections (tuberculosis, NCC), remote symptomatic due to TBI, previous stroke, CNS tumors and undetermined; this data is compatible with the mixed epidemiological profile of developed and developing countries expected in our country. ${ }^{6,27-29}$ The results from the present study were similar, except for CNS infections, as only three patients had demonstrated infectious etiologies and only two of them had NCC. It is possible that among the patients who did not have all the necessary diagnostic tests performed on them (27.1\% from total), there could have been cases of infectious etiology like undiagnosed $\mathrm{NCC}^{5,27-29}$ It is worth noting that tests could not be performed in some cases due to patient reluctancy (e.g., lumbar puncture) and in others due to lack of resources (e.g., MRI, Western Blot for NCC, AED serum dosage, etc.). Additionally, when prior history of epilepsy exists, it is important to rule out other etiologies aside from AED suspension, especially the acute ones. ${ }^{5}$ This affirmation was evidenced in our study as almost half of the patients with epilepsy had an etiology different from AED suspension (undetermined, CNS infection, alcohol abstinence or chronic consumption, etc.).

\section{AED adherence, complications and Rankin Score}

AED suspension was present in three-quarters of the patients, which is in concordance with the reported literature..$^{30}$ Among the risk factors associated with such finding, we predominantly found the following factors in our cohort: male sex, unemployment and low educational level. ${ }^{13}$ One of the most frequent complications associated with GCSE in our study was intrahospital/aspiration pneumonia concurring with literature, where pneumonia is reported in 16\% of patients. ${ }^{21}$ Numerous studies in adults used the modified Rankin scale to define functionality in patients with SE at short, medium and long-term. ${ }^{31}$ In our study, patients with a Rankin score of 3-5 at discharge represented $28.8 \%$ from the total, which is within the range (20-50\%) of patients with a significant functional disability after SE reported in the literature. ${ }^{3}$ The low proportion of patient follow-up at 1 month after discharge could be due to those presenting lower Rankin score at discharge (lower functional disability), considering unnecessary to attend follow-up.

\section{STESS and mortality}

Finally, half of the patients obtained a STESS $<3$, a score validated to predict in-hospital mortality of SE. ${ }^{32}$ The majority of them could be regarded as individuals with a low probability of in-hospital death. In addition, AED suspension, the most common etiology in our study, is associated with low mortality. ${ }^{5}$ This data has been corroborated in our study because the mortality associated with GCSE was $8.47 \%$, which falls within the range reported in the literature $(3-40 \%)^{5}$ and is similar to previous Peruvian studies with $7.3 \%$ and $8.5 \%{ }^{27,28}$

Among the five patients who died in our cohort, factors associated with high mortality rates such as anoxia, advanced age, numerous comorbidities, mechanical ventilation and longer duration of seizures were present. Furthermore, all of them presented with intermittent GCSE without convulsions at arrival, which delayed their diagnosis and treatment. ${ }^{5,28}$

The limitations of this study, aside from those inherent to its observational nature, include the lack of registration of both the time from the convulsive seizure cessation to the recovery of consciousness and the duration of the stabilization phase. In addition, due to the small number of patients with CGCSE in comparison with the other clinical presentations, we considered that the differences found in variables aside from etiologies and time were not signfificant. We conclude that patients that did not present with convulsive seizures (IGCSE) at arrival registered higher times in general than those who did, very likely due to the difficulty diagnosing their non-convulsive phase as SE because of the lack of EEG. For them, there was an untimely diagnosis. All of the registered times differed from the ones recommended in the guidelines, possibly due to the untimely diagnosis (e.g., time to 1st line treatment), lack of EEG for ensuring cessation of the GCSE (e.g., time to 2nd line treatment) and/or unavailability of ICU beds (e.g., time to 3rd line treatment), which are all contributing factors for an untimely treatment. The most used AEDs as 1st, 2nd, and 3rd line of therapy were one BZD, phenytoin and additional doses of BZDS, respectively, which might be due to lack of knowledge and/or resources. In general, the drugs used were given in adequate doses. The most frequent etiologies were AEDs suspension, undetermined (probably because of lack of diagnostic tools) and acute stroke.

Topics beyond the scope of this article such as pre-hospital management, factors influencing the described times, AEDs adherence, NCSE prevalence and long-term outcomes should be further explored for a better understanding of SE in Peru. Based on these preliminary data, and according to literature, where EEG availability and training in the ED proved to be useful for identifying subclinical and nonconvulsive seizures, ${ }^{33,34}$ the authors highly recommend the development of a local management guideline that includes EEG invariably and $\mathrm{CEEG}$ when possible to diagnose and subsequently ensure the cessation of GCSE, particularly during its non-convulsive phase (IGCSE), plus serum dosage of phenytoin as it was the most 
used 2nd line AED.

\section{Conflict of Interest}

The authors declare that they have no conflicts of interest.

\section{References}

1. Trinka $E$, Cock $H$, Hesdorffer $D$, et al. A definition and classification of status epilepticus--report of the ILAE task force on classification of status epilepticus. Epilepsia 2015;56:1515-23.

2. Nelson SE, Varelas PN. Status epilepticus, refractory status epilepticus, and super-refractory status epilepticus. Continuum (Minneap Minn) 2018;24:1683-707.

3. Hirsch $\sqcup$, Gaspard N. Status epilepticus. Continuum (Minneap Minn) 2013;19:767-94.

4. Chin RF, Neville BG, Scott RC. A systematic review of the epidemiology of status epilepticus. Eur I Neurol 2004;11:800-10.

5. Drislane FW, Kaplan PW. Status Epilepticus: A Clinical Perspective. 2nd ed. Cham: Springer Nature, 2018.

6. Sánchez S, Rincon F. Status epilepticus: epidemiology and public health needs. J Clin Med 2016;5:71.

7. Betjemann JP, Lowenstein DH. Status epilepticus in adults. Lancet Neurol 2015;14:615-24.

8. Glauser T, Shinnar S, Gloss D, et al. Evidence-based guideline: treatment of convulsive status epilepticus in children and adults: report of the guideline committee of the American Epilepsy Society. Epilepsy Curr 2016;17:48-61.

9. Cheng JY. Latency to treatment of status epilepticus is associated with mortality and functional status. I Neurol Sci 2016;370:290-5.

10. Kämppi L. Delays in the Treatment of Status Epilepticus - Effect on Outcome. 1st ed. Helsinki: University of Helsinki, 2018.

11. Chin RF, Neville BG, Peckham C, Wade A, Bedford H, Scott RC. Treatment of community-onset, childhood convulsive status epilepticus: a prospective, population-based study. Lancet Neurol 2008;7:696-703.

12. Sánchez Fernández I, Gaínza-Lein $M$, Abend NS, et al. Factors associated with treatment delays in pediatric refractory convulsive status epilepticus. Neurology 2018;90:e1692-701.

13. Giraldo-Tapia RS, Tejero-Alcalde M, Urdániga-Alvarado JE, SamalvidesCuba F, Guillén-Pinto D. Conocimientos y creencias acerca de epilepsia en los padres de familia usuarios habituales del centro de salud de Peralvillo-Huaral. Rev Neuropsiquiatr 2013;76:42.

14. Murthy JM, Jayalaxmi SS, Kanikannan MA. Convulsive status epilepticus: clinical profile in a developing country. Epilepsia 2007;48:2217-23.

15. Hill $C E$, Parikh $A O$, Ellis $C$, Myers JS, Litt $B$. Timing is everything: where status epilepticus treatment fails. Ann Neurol 2017;82:155-65.

16. Nagayama $M$, Yang $S$, Geocadin $R G$, et al. Novel clinical features of nonconvulsive status epilepticus. F1000Res 2017;1:1690.

17. Claassen J, Hirsch L. Refractory status epilepticus. In: Sirven JI, Stern
$\mathrm{JM}$, ed. Atlas of video-EEG monitoring. 1st ed. New York: McGraw-Hill, 2011;225-38.

18. Claassen J, Taccone FS, Horn P, et al. Recommendations on the use of EEG monitoring in critically ill patients: consensus statement from the neurointensive care section of the ESICM. Intensive Care Med 2013;39:1337-51.

19. Yigit O, Eray O, Mihci E, Yilmaz D, Arslan S, Eray B. The utility of EEG in the emergency department. Emerg Med J 2012;29:301-5.

20. Abdel Baki SG, Omurtag A, Fenton AA, Zehtabchi S. The new wave: time to bring EEG to the emergency department. Int / Emerg Med 2011;4:36.

21. Sutter R, Dittrich T, Semmlack S, Rüegg S, Marsch S, Kaplan PW. Acute systemic complications of convulsive status epilepticus-a systematic review. Crit Care Med 2017;46:138-45.

22. Tatishvili N, et al. El manejo del estado epiléptico infantil. Práctica Clínica Nacional. Recomendaciones (lineamientos) y enfermedades 2017;1-11.

23. Brophy GM, Bell R, Claassen J, et al. Guidelines for the evaluation and management of status epilepticus; Neurocrit Care 2012;17:3-23.

24. Aranda A, Foucart G, Ducassé JL, Grolleau S, McGonigal A, Valton L. Generalized convulsive status epilepticus management in adults: a cohort study with evaluation of professional practice. Epilepsia 2010;51:2159-67.

25. Skinner HJ, Dubon-Murcia SA, Thompson AR, et al. Adult convulsive status epilepticus in the developing country of Honduras. Seizure 2010; 19:363-7.

26. Parke-Davis Div of Pfizer Inc. DILANTIN-125- phenytoin suspension [Internet]. Silver Spring (MD): U.S. Food and Drug Administration [cited 2019 Mar 1]. Available at : http://labeling.pfizer.com/showlabeling. aspx?id=560.

27. Maldonado A, Ramos W, Pérez J, Huamán LA, Gutiérrez EL. Convulsive status epilepticus: clinico-epidemiologic characteristics and risk factors in Peru. Neurologia 2010;25:478-84.

28. Graus-Tejeda JM, Huerto-Aguilar JL, Macavilca-Cruz MA, Nájar-Trujillo NE, Rodríguez-Hurtado D. Clinical and epidemiological factors related with mortality in patients with status epilepticus in a general hospital in Lima: a comparative case series. Rev Neuropsiquiatr 2016;79:207-15.

29. De la Cruz WF, Zapata WR, Delgado JC, Mija L. Convulsive status epilepticus in adults attending the Instituto Nacional de Ciencias Neurológicas in Lima, Perú 2011-2013. Rev Neuropsiquiatr 2014;77: 236-41.

30. Malek N, Heath CA, Greene J. A review of medication adherence in people with epilepsy. Acta Neurol Scand 2017;135:507-15.

31. Sculier C, Gaínza-Lein M, Sánchez Fernández I, Loddenkemper T. Longterm outcomes of status epilepticus: a critical assessment. Epilepsia 2018;59(Suppl Suppl 2):155-69.

32. Wang $P$, Guan $Y$, Lin $X$, et al. Diagnostic value of status epilepticus severity score for survival conditions of patients with status epilepticus: a PRISMA-compliant systematic review and meta-analysis. Epilepsy Behav 2018;82:104-10.

33. Máñez Miró JU, Díaz de Terán FJ, Alonso Singer P, Aguilar-Amat Prior 
92 Journal of Epilepsy Research Vol. 11, No. 1, 2021

MJ. Emergency electroencephalogram: usefulness in the diagnosis of nonconvulsive status epilepticus by the on-call neurologist. Neurologia (Engl Ed) 2018:33:71-7.
34. Chari G, Yadav K, Nishijima D, Omurtag A, Zehtabchi S. Improving the ability of ED physicians to identify subclinical/electrographic seizures on EEG after a brief training module. Int J Emerg Med 2012;12:11. 